Revue d'histoire de l'Amérique française

ZH REVUE D.HISTOIRE DE L'AMÉRIQUE FRANÇAISE

\title{
Les groupes financiers et la guerre du papier au Canada,
} 1920-1930

\section{Gilles Piédalue}

Volume 30, numéro 2, septembre 1976

URI : https://id.erudit.org/iderudit/303529ar

DOI : https://doi.org/10.7202/303529ar

Aller au sommaire du numéro

\section{Éditeur(s)}

Institut d'histoire de l'Amérique française

\section{ISSN}

0035-2357 (imprimé)

1492-1383 (numérique)

Découvrir la revue

Citer cet article

Piédalue, G. (1976). Les groupes financiers et la guerre du papier au Canada, 1920-1930. Revue d'histoire de l'Amérique française, 30(2), 223-258.

https://doi.org/10.7202/303529ar d'utilisation que vous pouvez consulter en ligne.

https://apropos.erudit.org/fr/usagers/politique-dutilisation/ 


\section{LES GROUPES FINANCIERS \\ ET \\ LA GUERRE DU PAPIER AU CANADA \\ 1920-1930*}

Gili es Piédai ue

Département d'histoire

Univ'ersité de Montréal

Cet article fait suite à une étude préliminaire sur les groupes financiers au Canada entre 1900 et 1930 , étude que j'ai récemment publiée dans cette revue ${ }^{1}$. Cette étude montrait essentiellement qu'un groupe de plus en plus restreint de personnes dirigeait les principales sociétés au Canada durant le premier tiers du vingtième siècle. Bien plus, ce groupe, qu'on allait appeler grande bourgeoisie, était secoué par des rivalités internes. En effet, l'utilisation de la méthode des administrateurs communs faisait apparaître clairement des factions à l'intérieur d'une grande bourgeoisie apparemment unie. Le groupe de la Banque de Montréal, ceux de la Banque Canadienne de Commerce et de la Banque Royale formaient les principales fractions. Par le jeu des alliances ou par une lutte plus ou moins ouverte, chaque groupe tentait d'affirmer ou de maintenir sa prépondérance et son leadership dans les principaux secteurs de

\footnotetext{
* Je tiens à remercier Messieurs Alfred Dubuc et Paul-André Linteau, tous deux professeurs au département d'histoire de l'Université du Québec à Montréal, de leur support et de leurs conseils éclairés. Je veux souligner de façon toute particulière l'aide du professeur Dubuc qui a bien voulu m'ouvrir les archives du projet de recherche qu'il dirige sur l'histoire de la société Consolidated-Bathurst Ltd.

1 Gilles Piédalue, «Les groupes financiers au Canada, 1900-1930, une étude préliminaire", Revue d'histoire de l'Amérique française, 30, no 1 (juin 1976) : 3-34.

[ 223]
}

RHAF, vol. 30, $\mathrm{n}^{\circ} 2$ (septembre 1976) 
l'activité économique. Durant les années 1920, le groupe de la Banque Royale dominait le monde des affaires au pays. Lié étroitement au groupe de la Banque de Montréal, il luttait âprement pour maintenir sa position privilégiée dans plus d'un secteur. C'est l'histoire d'une de ces luttes que nous allons relater ici, celle de la guerre du papier. Celle-ci opposait les papeteries du groupe de la Banque Royale à celles de l'International Paper Co. directement liées aux grands éditeurs nord-américains. Ce conflit prenait en apparence l'aspect d'un conflit entre la grande bourgeoisie canadienne et la grande bourgeoisie américaine. Il faut toutefois nuancer ce portrait global en constatant que chacune des deux factions est formée d'une alliance entre des groupes canadiens et américains. Le conflit débordait largement le cadre canadien et il avait pour enjeu le contrôle du prix du papier journal en Amérique du Nord.

Le présent exposé se divise en trois sections principales. D'abord je brosserai un tableau de la situation dramatique dans laquelle se trouvait l'industrie canadienne du papier après 1920. J'y décrirai aussi les principaux correctifs apportés par le puissant groupe de la Banque Royale pour réorganiser cette activité. L'utilisation de la concentration comme principal remède déclenchait toute une série de réactions. Dans la deuxième section, j'analyserai les réactions du groupe formé par l'International Power \& Paper Co. et les grands journaux nord-américains aux tentatives de formation d'un cartel du papier dirigé par le groupe de la Banque Royale. Enfin, en dernière partie, nous verrons comment un conflit plus ou moins larvé dégénéra en une guerre ouverte entre les principales factions en présence à la fin des années 1920.

\section{Le groupe de la Banque Royale et le mouvement de concentration, 1923-1930}

La première guerre mondiale avait été une période de très grande prospérité dans le secteur des pâtes et papiers au Canada. De 1915 à 1920 , le prix du papier journal passait de $\$ 41.60$ à $\$ 114.70$ la tonne (voir figure 1). Malgré des investissements massifs de 143 millions de dollars au cours de cette période (voir tableau 1), le taux de profit sur le stock de capital investi représentait près de $40 \%$ en 1920 , un sommet inégalé depuis 1880 (voir tableau 2). Mais la crise de 1921 mettait bientôt fin à cette prospérité du temps de guerre. En effet, le prix du papier journal tombait à $\$ 77.20$ la tonne de 1921 à 1922. Après un bref redressement, il amorçait une chute vertigineuse 


\section{FIGURE 1}

La courbe de l'évolution du prix de la tonne de papier journal, 1914-1950 (en dollars américains)

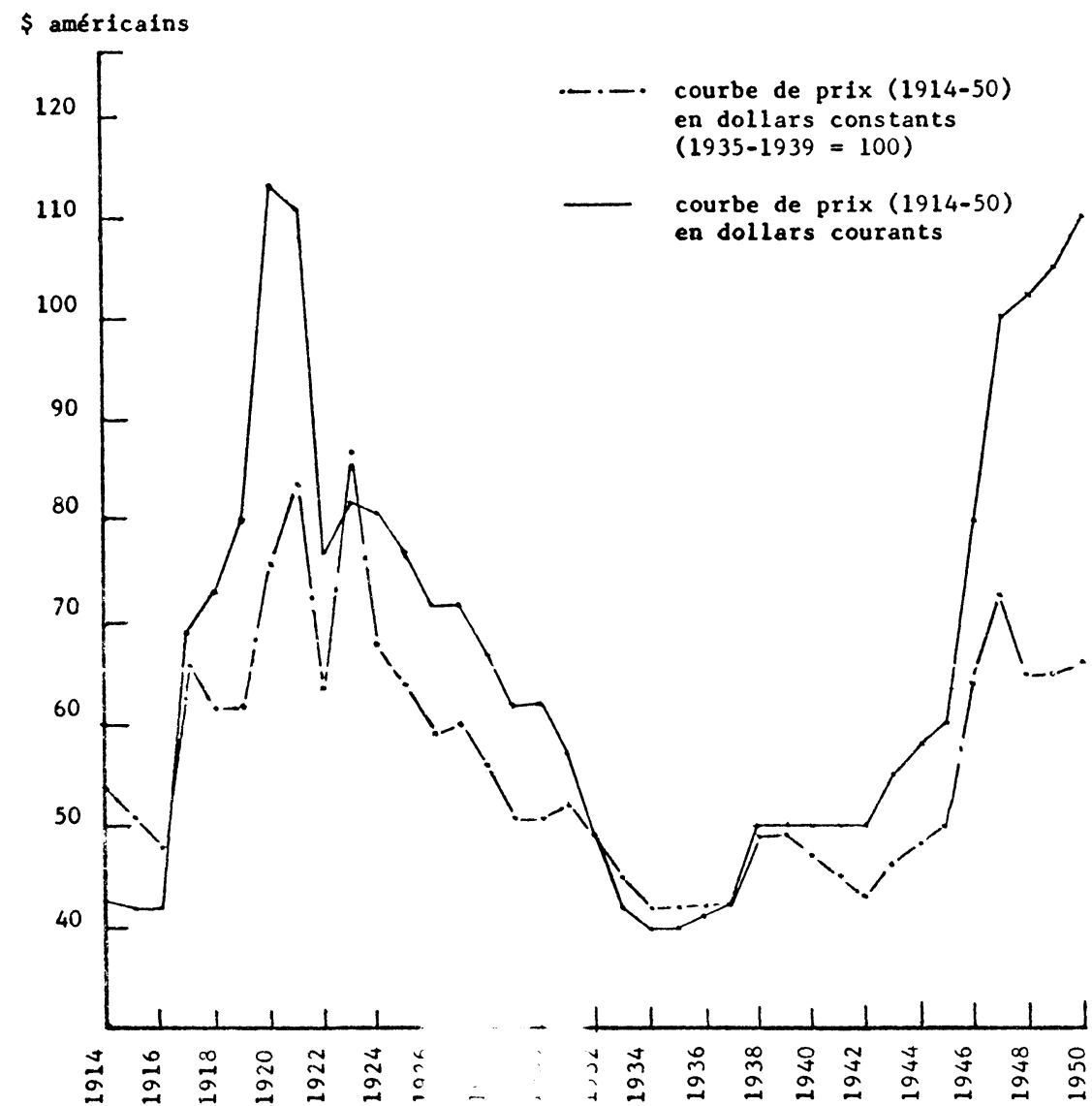

Sources: J.A. Guthrie, The newsprint paper industry, an economic analysis (Cambridge, Harvard University Press, 1941), 247-48. - Association Canadienne des Producteurs de Pâtes et Papiers, Annual newsprint supplement (avril 1969): tableau 26, 11. M.C. Urquhart, et K.A.H. Buckley, Historical statistics of Canada (Toronto, The Macmillan Co. of Canada Ltd, 1965), 304. 
TABLEAU 1

Industrie canadienne des pâtes et papiers, stock total de capital fixe (en milliers de dollars courants), 1870-1934

$\begin{array}{rrrr}1870 & 500 & 1922 & 303800 \\ 1880 & 1600 & 1923 & 344300 \\ 1890 & 5200 & 1924 & 373500 \\ 1900 & 15300 & 1925 & 369900 \\ 1905 & 20100 & 1926 & 405000 \\ 1910 & 40000 & 1927 & 470500 \\ 1915 & 115600 & 1928 & 539400 \\ 1917 & 153900 & 1929 & 537900 \\ 1918 & 188000 & 1930 & 595400 \\ 1919 & 206100 & 1931 & 536300 \\ 1920 & 258200 & 1932 & 528900 \\ 1921 & 288000 & 1933 & 500500 \\ & & 1934 & 495000\end{array}$

Source: Canada, Dominion Bureau of Statistics, Fixed capital flows and stocks, manufacturing industries, 1926-1960, 65 (catalogue number 13-522). Le stock de capital fixe est vraisemblablement net de dépréciation et englobe la valeur des propriétés foncières, de la machinerie et des installations.

passant de $\$ 81.80$ à $\$ 40.00$ la tonne de 1923 à 1934. Parallèlement à cette chute des prix, le stock de capital investi dans le secteur doublait presque. La capacité canadienne de production passait de 1.8 à 4 millions de tonnes par année de 1925 à 1930) pour enfin se stabiliser à ce dernier niveau de 1930 à 1934. D'autre part les importations de son principal client, les États-Unis, grimpaient de 1.45 à 2.4 millions de tonnes par an de 1925 à 1929, pour tornber à 1.8 million de tonnes en 1933. ${ }^{2}$ Ces investissements étaient démesurés, sans proportion avec la capacité d'absorption du marché américain. La situation devenait d'autant plus critique que ce marché consommait près de $80 \%$ de la production canadienne de 1914 à 1927 (voir figure 2). À la

2 Canada, Chambre des Communes, Comité permanent de la banque et du commerce, délibérations (6 mars au 14 juin 1934): 917 (à l'avenir: Comité permanent de la banque, 1934). 
FIGURE 2

Production canadienne de papier journal (1914-1936)

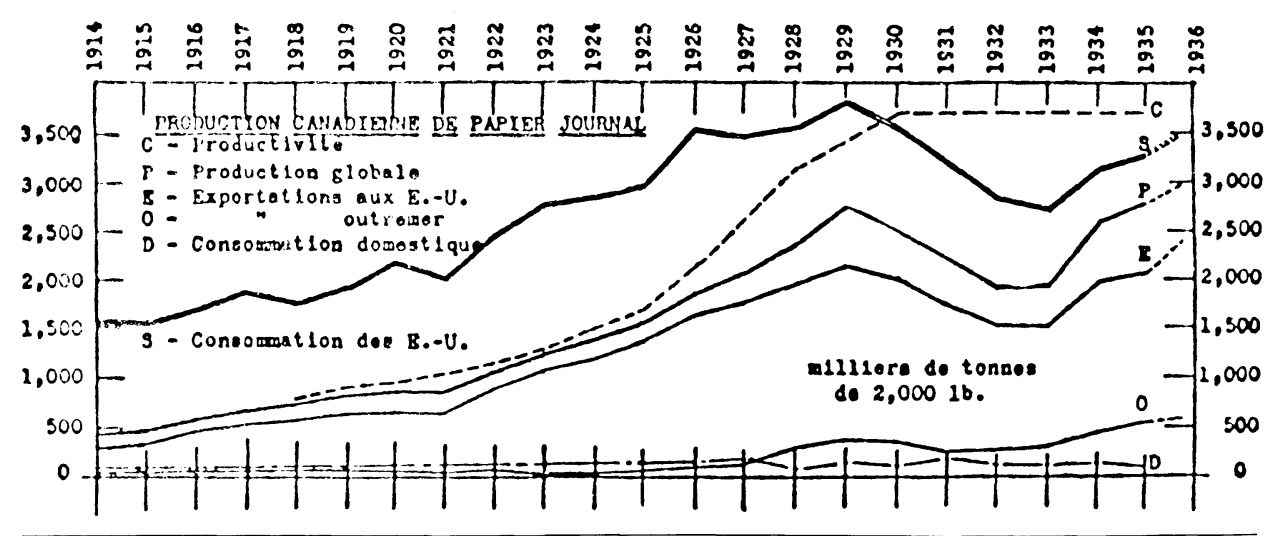

Source: P.-E. Piché, «Où en est notre industrie du papier», Actualité économique, XII, tome II, 1 (novembre 1936): 19. 
TABLEAU 2

Industric cancadienne des pâtes et papiers, coluts de production, profits (en milliers de dollars courants) et taux de profit 1870-1933

\begin{tabular}{|c|c|c|c|c|c|c|}
\hline & $\begin{array}{l}\text { Valeur } \\
\text { brute de la } \\
\text { production } \\
\text { (1) }\end{array}$ & $\begin{array}{c}\text { Coût des } \\
\text { matières } \\
\text { premières } \\
\text { utilisées } \\
\text { (2) }\end{array}$ & $\begin{array}{c}\text { Salaires } \\
\text { et gages } \\
\text { (3) }\end{array}$ & $\begin{array}{c}\text { Coûts en } \\
\text { carburant et } \\
\text { électricité } \\
\text { (4) }\end{array}$ & $\begin{array}{l}\text { Profits Nets } \\
\text { (1) }-(2+3+4)\end{array}$ & $\begin{array}{c}\text { Taux de } \\
\text { Profit }\end{array}$ \\
\hline 1870 & 1725 & 874 & 295 & 45 & 511 & 102.20 \\
\hline 80 & 2976 & 1628 & 576 & 62 & 710 & 44.37 \\
\hline 90 & 6206 & 3160 & 1455 & 129 & 1462 & 28.11 \\
\hline 1900 & 12420 & 5416 & 3347 & 297 & 3360 & 21.96 \\
\hline 05 & 18761 & 10446 & 4685 & 615 & 6962 & 34.63 \\
\hline 10 & 32999 & 15477 & 7626 & 804 & 9092 & 22.73 \\
\hline 15 & 76684 & 30592 & 16538 & 2051 & 23177 & 20.04 \\
\hline 17 & 120370 & 45707 & 25450 & 8652 & 40561 & 26.36 \\
\hline 18 & 143880 & 56920 & 32079 & 12385 & 42495 & 22.60 \\
\hline 19 & 169840 & 68412 & 38841 & 1237.5 & 50211 & 24.36 \\
\hline 1920 & 278728 & 106074 & 53559 & 1681.4 & 102281 & 39.61 \\
\hline 21 & 179167 & 75382 & 40746 & 1536.4 & 47675 & 16.55 \\
\hline 22 & 188074 & 78055 & 39736 & 16173 & 54110 & 17.81 \\
\hline 23 & 216176 & 86795 & 45825 & 1865.4 & 64902 & 18.85 \\
\hline 24 & 207790 & 86861 & 44772 & 17775 & 58381 & 15.63 \\
\hline 25 & 222615 & 91815 & 45431 & 18175 & 67193 & 18.17 \\
\hline 26 & 248831 & 102066 & 51994 & $2079 ?$ & 73979 & 18.27 \\
\hline 27 & 259205 & 105275 & 54489 & 24445 & 74996 & 15.94 \\
\hline 28 & 277346 & 111373 & 56732 & 2497.4 & 84267 & 15.62 \\
\hline 29 & 290950 & 121401 & 60622 & 25829 & 83098 & 15.45 \\
\hline 1930 & 261621 & 104975 & 55918 & 26655 & 74073 & 12.44 \\
\hline 31 & 217250 & 85053 & 45292 & 23414 & 63491 & 11.84 \\
\hline 32 & 169368 & 65500 & 36560 & 20299 & 47010 & 8.89 \\
\hline 33 & 157509 & 64895 & 34925 & 19383 & 38301 & 7.65 \\
\hline
\end{tabular}

Source: M.C. Urquhart et K.A.H. Buckley, Historical Statistics of Canada (Toronto, Macmillan Co. of Canada Ltd., 1965), 470. De plus la donnée de la colonne (1) pour 1915, celles de la colonne: (2) pour 1905 et 1915 ainsi que celle de la colonne (3) pour 1915 sont des estimés uniquement. Les données de la colonne (4) pour 1870, 1880,1890,1900,1905, 1910 et 1915 sont des estimés calculés à partir de la moyenne de $8.13 \%$, représentant le pourcentage moyen des coûts en énergie par rapport aux profits bruts de 1917 à 1927 inclusivement. De plus les données de 1917 à 1943 sont obtenues en soustrayant de la valeur brute de production le coût des matières premières et la valeur ajoutée de production. 
recherche de nouveaux marchés, les producteurs canadiens réussissaient malgré tout de 1927 à 1929 à augmenter le niveau de leurs exportations outre-mer. Celles-ci passaient de 200,000 à 400,000 tonnes. La crise de 1929 allait malheureusement mettre un terme à cet effort de diversification.

Le jeu combiné de la baisse du prix du papier journal et de l'investissement massif entraînait inévitablement la chute du taux de profit. Entre 1920 et 1931 , celui-ci tombait de 40 à 12\% (voir tableau 2). Devant cette détérioration rapide de la situation une solution s'imposait: la concentration des sociétés papetières. Elle devenait le seul moyen de maintenir le taux de profit en contingentant la production et en fixant les prix. La tentative de concentration des papeteries fut menée principalement par le groupe financier de la Banque Royale du Canada entre 1923 et 1930.

L'industrie canadienne des pâtes et papiers avait déjà connu depuis 1900 deux mouvements de concentration, l'un de 1908 à 1913 et l'autre de 1915 à 1920. Mais celui de 1923 à 1930 était d'une intensité inégalée jusque-là. Dans les deux premiers mouvements, 27 sociétés, représentant un actif total de $\$ 60.9$ millions, avaient été absorbées. Par contre, le dernier mouvement de concentration touchait 58 sociétés totalisant $\$ 440$ millions (voir tableau 3). Ces trois mouvements étaient la réaction logique à la baisse du taux de profit dans cette industrie depuis 1870. Celui-ci s'était en effet abaissé de 102 à $12.44 \%$ entre 1870 et 1930 (voir tableau 2). Notons en passant que si les divers mouvements de concentration s'inscrivent dans le cadre général de la baisse du taux de profit, ils atteignent habituellement une intensité maximum en période de hausse de l'indice du prix des actions ordinaires (voir tableau 3). Les opérations de concentration deviennent alors des plus lucratives pour les promoteurs.

La baisse du taux de profit que nous avons observée n'était pas unique au secteur des pâtes et papiers. Elle touchait tous les secteurs industriels et entraînait, de 1900 à 1930, la formation de groupes financiers de plus en plus denses. Comme nous l'avons déjà souligné, c'est le groupe financier de la Banque Royale qui dirigeait principalement le vaste mouvement de concentration de 1923 à 1930 . Ce groupe, en formation depuis 1901 et en expansion rapide à partir de 1910, s'imposait alors comme le plus puissant au Canada. Il avait provoqué entre 1910 et 1923 l'éclatement du bloc formé par le groupe de la Banque de Montréal et le groupe de la Banque 


\section{TABLEAU 3}

\section{Industrie canadienne des pâtes et papiers, mouvements de concentration 1900-1933}

nombre de concentrations (consolidations) nombre de sociétés absorbées actif total des concentrations (consolidations) (en milliers de \$) indice du prix des actions ordinaires $(1900-48=100)$

\begin{tabular}{|c|c|c|c|c|}
\hline 1900 & & & & 67.5 \\
\hline 01 & & & & 71.3 \\
\hline 02 & & & & 75.5 \\
\hline 03 & & & & 70.9 \\
\hline 04 & & & & 67.2 \\
\hline 05 & & & & 73.4 \\
\hline 06 & 1 & 1 & 100 & 77.0 \\
\hline 07 & & & & 71.3 \\
\hline 08 & 1 & 1 & 1000 & 69.5 \\
\hline 09 & 1 & 2 & 1000 & 78.1 \\
\hline 1910 & 2 & 2 & 700 & 81.7 \\
\hline 11 & 2 & 5 & 2700 & 85.9 \\
\hline 12 & 1 & 1 & 3000 & 90.3 \\
\hline 13 & 2 & 5 & 14100 & 87.0 \\
\hline 14 & & & & 72.9 \\
\hline 15 & 1 & 1 & 4000 & 67.5 \\
\hline 16 & 1 & 1 & 900 & 76.3 \\
\hline 17 & 1 & 3 & 5500 & 72.6 \\
\hline 18 & 1 & 1 & 4000 & 69.5 \\
\hline 19 & 1 & 1 & 1600 & 78.3 \\
\hline 1920 & 4 & 4 & 22400 & 79.3 \\
\hline 21 & & & & 69.1 \\
\hline 22 & & & & 74.7 \\
\hline 23 & 1 & 1 & 100 & 81.8 \\
\hline 24 & 2 & 2 & 11000 & 84.2 \\
\hline 25 & 7 & 7 & 107100 & 96.2 \\
\hline 26 & 7 & 8 & 22300 & 118.8 \\
\hline 27 & 6 & 12 & 70300 & 147.3 \\
\hline 28 & 7 & 12 & 86800 & 190.3 \\
\hline 29 & 7 & 12 & 78500 & 227.5 \\
\hline 1930 & 3 & 4 & 63900 & 162.4 \\
\hline 31 & & & & 101.7 \\
\hline 32 & 3 & 3 & 2500 & 66.2 \\
\hline 33 & & & & 81.8 \\
\hline
\end{tabular}

Source: J.C. Weldon, Consolidations in Canadian Industry, 1900-1948, étude préparée pour la Combines Investigation Commission (Ottawa, 1948), 29, 34. 
Canadienne de Commerce et s'était substitué à ce dernier dans l'ancienne alliance. Entre 1923 et 1930, l'alliance entre le groupe de la Banque Royale et celui de la Banque de Montréal se solidifiait tandis que le groupe de la Banque Canadienne de Commerce se séparait de plus en plus du bloc principal ${ }^{3}$.

La tentative de formation d'un cartel canadien du papier journal passait par un rapprochement de plus en plus étroit entre les sociétés papetières et le groupe de la Banque Royale et un réseau très dense de liens personnels se tissait entre les conseils d'administration. Le nombre de sociétés papetières liées par au moins un administrateur commun aux diverses sociétés du groupe de la Banque Royale passait de 11 à 15 entre 1923 et 1930 (voir tableaux 4 et 5). Le montant des prêts accordés par la Banque Royale aux sociétés papetières atteignait $\$ 31$ millions en 1931 comparativement à $\$ 5$ millions en $1926^{4}$.

La Banque Royale favorisait ouvertement la concentration des sociétés papetières canadiennes. Par l'intermédiaire des sociétés de placement Holt, Gundy \& Co. et Wood, Gundy \& Co., elle créait progressivement Canada Power \& Paper Corporation de 1923 à 1930. H.S. Holt, le président de la Banque Royale de 1908 à 1930, était l'associé de J.H. Gundy dans la société de placement Holt, Gundy $\&$ Co., dans laquelle il détenait $30 \%$ des intérêts ${ }^{5}$. Pour sa part, J.H. Gundy, membre de la société de placement Wood, Gundy \& Co., déclarait faire la majeure partie de ses transactions par l'entremise de cette société ${ }^{6}$. De plus, la Banque Royale avait consenti à ces deux sociétés de placement des prêts importants de 1926 à 1933. En 1927 par exemple les prêts accordés à Holt, Gundy \& Co. atteignaient $\$ 1,695,030$. et ceux consentis à Wood, Gundy \& Co. se chif-

3 Gilles Piédalue, «Les groupes financiers... », RHAF, ibid.: 22.

4 Comité permanent de la banque, 1934: 767.

Prêts de la Banque Royale aux sociétés papetières (au ler décembre):

1926: $\$ 5,818,200$.

1928: $\$ 11,637,929$.

1927: $\$ 8,676,860$.

1930: $\$ 25,138,019$.

1929: $\$ 18,891,572$.

1933: $\$ 19,281,190$.

1931: $\$ 31,096,826$.

(Ici la valeur des prêts représente un stock).

5 Comité permanent de la banque, 1934: 780.

6 Ibid. : 656. 


\section{TABLEAU 4}

Administrateurs communs entre les 20 sociétés papetières canadiennes les plus importantes et les sociétés formant les principaux éléments des 3 grands groupes financiers canadiens (1923)

(A) groupe de la Banque de Montréal B) groupe de la Banque Royale

C) groupe de la Banque Canadienne de Commerce)

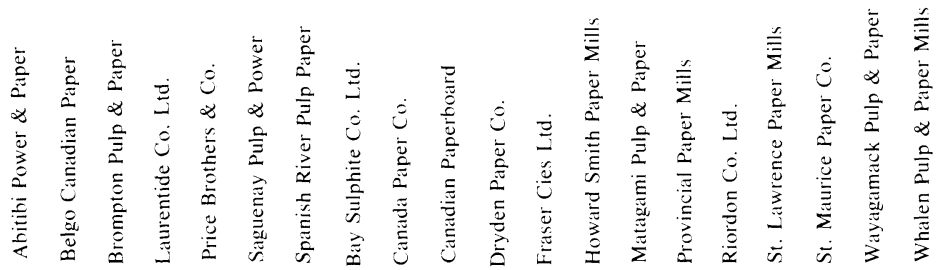

Banque de Montréal

Royal Trust Co.

Consol Mining \& Smelting Co.

C.P.R.

Québec Power Co.

Laurentide Power Co.

Shaw. Water \& Power Co.

Mackay Cies Ltd

Sun Life Ass. Co.

$\mathrm{Mtl}$ Light Heat \& Power Co.

Ogilvie Flour Mills

Canadian Cottons Ltd

Banque Royale

Montreal Trust $\mathrm{Co}$.

Canada Steamship Lines Ltd

Mont Royal Hotel Co.

Steel Co. of Canada

National Trust Co.

Winnipeg Electric Co. 
fraient à $\$ 6,989,330^{7}$. La Banque Royale avait aussi développé durant la même période une politique de prêts aux sociétés papetières dont H.S. Holt était administrateur ${ }^{8}$. La valeur de ces prêts s'élevait en moyenne à $\$ 5$ millions par année pour la période allant du premier décembre 1926 au premier décembre 1932.

La formation de Canada Power \& Paper Corporation s'effectua en trois phases. La première débutait en 1923 pour se terminer en 1925 avec la constitution de St. Maurice Valley Corporation. Cette

7 Ibid. : 780-781.

Prêts de la Banque Royale aux deux sociétés de placements (au ler décembre):

Holt, Gundy \& Cie

1926 \$ 392,292

$1927 \$ 1,695,030$

1928 nil

1929 nil

1930 nil

1931 nil

1932 nil

(ici la valeur des prêts représente un stock).

8 Ibid. : 771.

Prêts de la Banque Royale aux sociétés papetières dont H.S. Holt était administrateur de 1926 à 1933 (au 1 er décembre):

1926 St. Maurice Valley Corp.

Fort William Paper Co. Ltd

Price Brothers Co. Ltd

$\$ 2,000,000$

$\$ 2,630,477$

$\$ 6,989,330$

$\$ 959.442$

$\$ 4,162,180$

$\$ 3,288,058$

$\$ 4,705,848$

$\$ 715,807$

$\$ 2,675,757$
Anticosti Corporation Ittd

1927 Anticosti Corporation Ltd

Price Bros. \& Co. Ltd

1928 Anticosti Corporation Ltd

St. Maurice Valley Corp.

Price Bros. \& Co. Ltd

1929 St. Maurice Valley Corp.

Price Bros. \& Co. Ltd

Howard Smith Paper Mills Ltd

1930 Canada Power \& Paper Corp.

Price Bros. \& Co. Ltd

Howard Smith Paper Mills Ltd

1931 Canada Power \& Paper Corp.

Price Bros. \& Co. Ltd

Howard Smith Paper Mills Ltd

1932 Howard Smith Paper Mills Ltd (ici la valeur des prêts représente un stock).
100

240,000

$\$ 2,240,000$

$\$ 1,200,000$

$1,989,000$

$\$ 3,189,500$

$\$ 800,000$

$3,175,000$

$1,771,000$

$\$ 5,746,000$

$\$ 1,125,000$

$\$ 1,747,643$

$1,463,343$

$\$ 4,335,986$

$\$ 7,347,000$

$1,550,000$

1,252,915

$\$ 10,149,915$

$\$ 6,959,530$

$2,152,500$

$1,150,467$

$\$ 465,069$

$\$ 10,262,497$ 


\section{TABLEAU 5}

Administrateurs communs entre les sociétés papetières canadiennes les plus importantes et les sociétés

formant les éléments des 4 grands groupes financiers canadiens en 1930.

Banque de Montréal Dominion Textile Co.

Montreal Cottons $\mathrm{Co}$.

Dominion Rubber Co.

Ogilvie Flour Mills Co.

Canadian Industries Ltd.

Bell Telephone Co.

Royal Trust Co.

Banque Royale

Sun Life Ass. Co.

Montreal Trust Co.

Wabasso Cotton Co.

Canada Cement $\mathrm{Co}$.

Steel Co. of Canada

Dominion Steel \& Coal Co.

Nova Scotia Steel \& Coal Co.

Dominion Bridge Co.

Aluminium Ltd.

Canadian General Electric Co.

Shawinigan W. \& P. Co.

Duke-Price Power Co.

Montreal L.H. \& P. Consol.
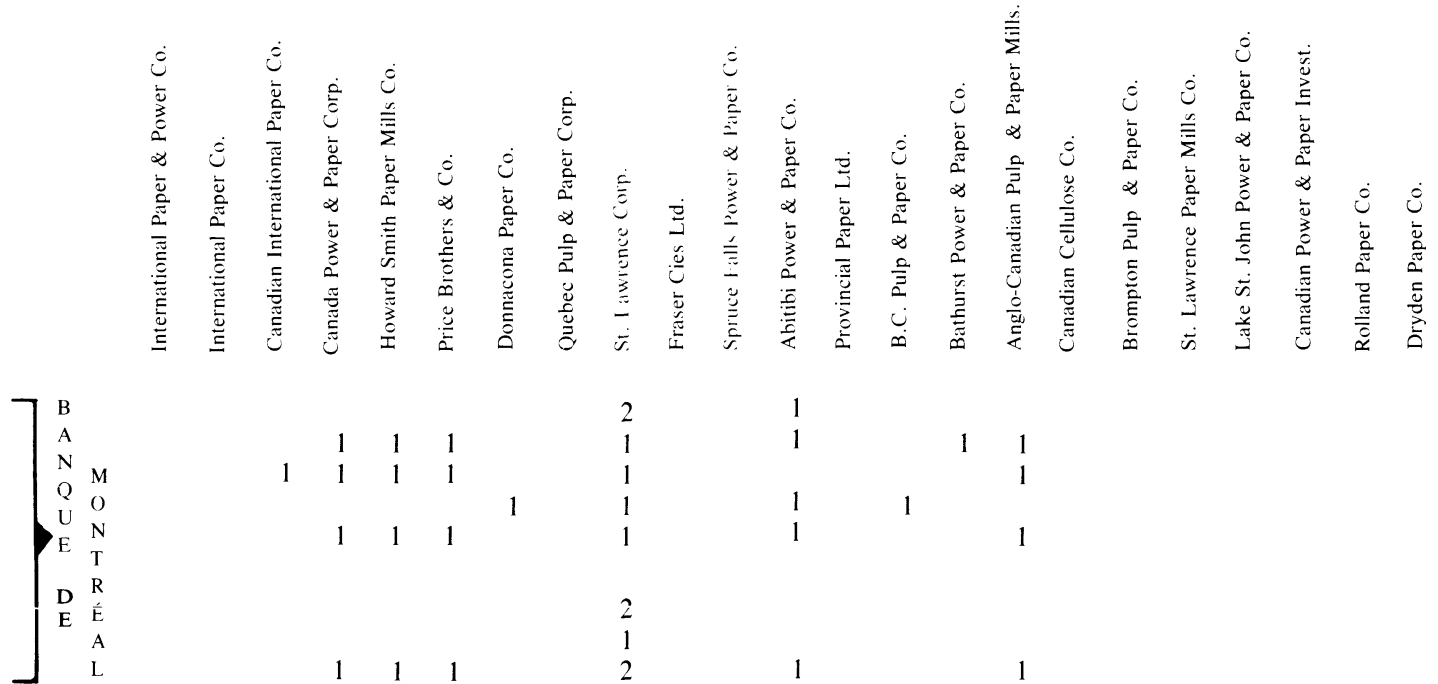

$$
\begin{aligned}
& 2 \\
& 1 \\
& 1 \\
& 1 \\
& 1
\end{aligned}
$$

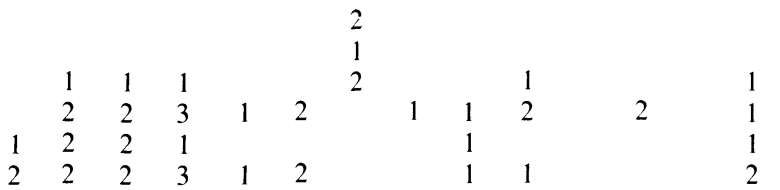

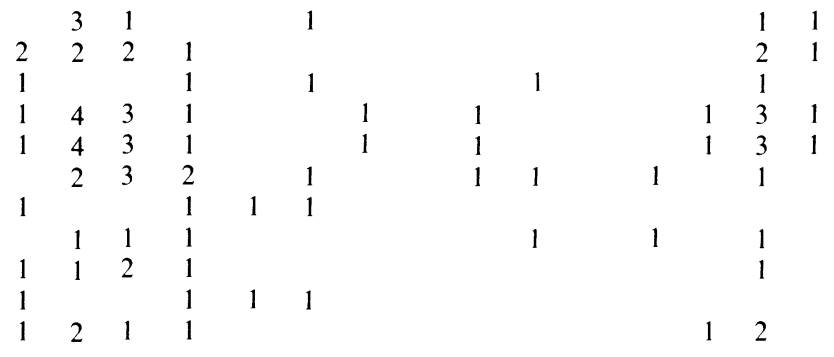


Consol. Mining \& S. Co. United Securities Ltd

Montreal Tramways Co.

C.P.R.

Can. Steamship Lines

Banque Can. de Com.

Canada Life Ass. Co.

Imperial Life Ass. Co.

Central Can. Loan \& S. Co.

National Trust Co.

Lake of the Woods Milling

Massey-Harris Co.

International H.-Elec. S.

Can. H.-Elec. Corp.

Mc Coll. Front. Oil Co.

Winnipeg Elec. Co.

Manitoba Power Co.

B.C. Power Corp.

Power Corp. of Can.

Can. Power Northern Co.

East Kootenay P. Co.

Southern Can. Power Co.

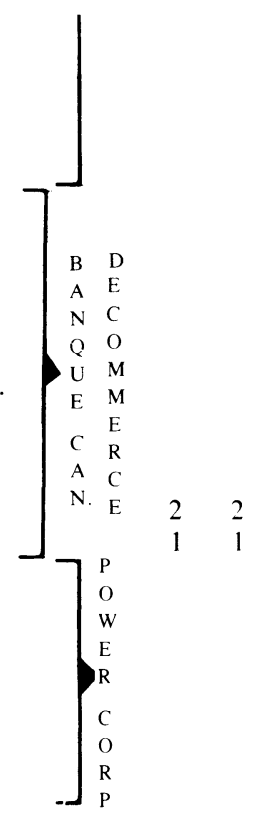

$\begin{array}{lll} & 1 & 1 \\ 1 & 1 & \\ 1 & 1 & 1 \\ & 1 & 1 \\ 2 & 2 & 1 \\ 4 & & \end{array}$

111

21

11

4

1

1

$\begin{array}{lll}2 & 2 & 1\end{array}$

1
1

1

1

$\begin{array}{ll}1 & 2\end{array}$

1

1

1

1

1

1

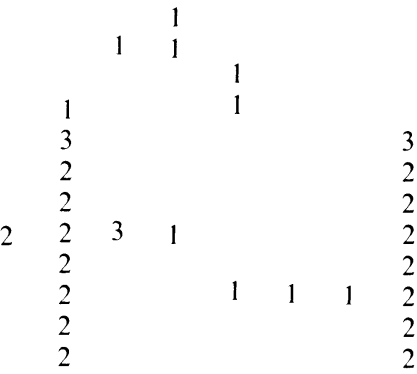

Sources: Annual Financial Review (Canadian) (Toronto Houston's Standard Publications) - annuel à partir de 1900Survey of Corporate Securities (Montreal, Financial Post) - annuel à partir de 1928 -. Sociétés papetières liées à aucun groupe: Mersey Paper Co. Superior Paper Mills Co. Western Québec Paper Mills Co. Interlake Tissue Mills Co., Clyde \& Sissiboo Pulp Co., Westminster Paper Co., Manitoba \& Ontario Paper Co. et St. Regis Paper Co. of Canada. 
société regroupait la Belgo Paper Co. de Shawinigan et la St. Maurice Paper Co. du Cap-de-la-Madeleine, une ancienne filiale de la société américaine Union Bag \& Paper Corporation. Puis de 1925 à 1927, la St. Maurice Valley Corporation se rapprochait de la Laurentide Co. Ltd. pour former en 1928 la Canada Power \& Paper Corporation. Finalement, Canada Power \& Paper Corporation absorbait successivement la Wayagamack Pulp \& Paper Co. et la Port Alfred Pulp \& Paper Corporation en 1929 ainsi que l'Anglo Canadian Pulp \& Paper Co. en 1930 (voir appendice). H.S. Holt et J.H. Gundy furent les principaux artisans de cette vaste opération échelonnée sur presque dix ans. On les retrouve constamment, directement impliqués dans le processus de concentration.

Membre du conseil d'administration de Belgo Paper Co. de 1923 à 1928, Holt en fut aussi vice-président de 1923 à 1926 et président de 1927 à 1928. Pour sa part J.H. Gundy, élu lui aussi administrateur de Belgo Paper Co. de 1923 à 1928, y occupa le poste de vice-président de 1927 à $1928^{\circ}$. De plus la société Wood, Gundy $\&$ Co. s'occupait dès 1923 de la vente d'une émission de $\$ 8,000,000$. en obligations de première hypothèque de la Belgo Paper Co. avec les sociétés de courtage Guaranty Co. et Newman, Sweezey \& Co. En janvier 1926, la Wood, Gundy \& Co. mettait seule en vente le dernier bloc de $\$ 1,200,000$. en obligations de première hypothèque de Belgo Paper Co. Ltd. La mise en marché des dernières actions privilégiées de Belgo, représentant un million de dollars, fut exécutée par la même société en février 1926. Dès 1925, le groupe Holt-Gundy acquérait un intérêt prépondérant dans Belgo Paper Co. ${ }^{10}$. La même année, J.H. Gundy prenait le contrôle de la St. Maurice Paper Co. dont il devenait admiristrateur. Il admettra un peu plus tard avoir joué un rôle important dans la formation de St. Maurice Valley Corporation ${ }^{11}$. Il en était élu administrateur et vice-président de 1925 à 1928. En décembre 1925, la société Wood, Gundy \& Co. se chargeait de la vente de $\$ 6,266,500$. en obligations de première hypothèque et $\$ 4,300,000$. en actions privilégiées de St. Maurice Valley Corporation. Cette dernière offrit aussi au public par l'entremise de la même société de courtage un autre bloc de $\$ 1,300,000$. en obligations de première hypothèque en juin 1926 . H.S. Holt occupa de 1925 à 1928 les postes d'administrateur et de

\footnotetext{
9 Annual Financial Review (Canadian) (Toronto, Houston's Standard Publications): 1924 à 1929 (à l'avenir: AFR).

10 Comité permanent de la banque 1934: 625.

11 Ibid. : 624.
} 
président à la St. Maurice Paper Co. et à la St. Maurice Valley Corporation. En 1926, C.B. McNaught devenait avec H.S. Holt le deuxième administrateur de la Banque Royale à la fois administrateur de St. Maurice Valley Corporation et de Consolidated Investment Corporation $^{12}$. Cette dernière, une société de placement, contrôlait St. Maurice Valley Corporation directement et indirectement par le biais d'une autre société de placement, la Newsprint Investment Corporation $^{13}$. Canada Paper Co. se joignait à St. Maurice Valley Corporation en 1926. Notons que depuis 1910, Holt siégait au conseil d'administration de Canada Paper Co. A.J. Brown, un autre administrateur de la Banque Royale, occupa le poste d'administrateur à l'Anticosti Corporation de 1926 à 1930. Cette société était la propriété commune de la St. Maurice Valley Corporation, de la Wayagamack Pulp \& Paper Co. et de la Port Alfred Pulp \& Paper Corporation.

Techniquement la formation de St. Maurice Valley Corporation se fit de la façon suivante. Dans un premier temps, le groupe Holt= Gundy acheta par l'entremise de Consolidated Investment Corporation les actifs de St. Maurice Paper à la société Union Bag \& Paper Corporation moyennant une participation de 31 1/4\% au capital-actions de Newsprint Investment Corporation ${ }^{14}$. Parallèlement, Consolidated Investment Corporation obtenait $85 \%$ du capital-actions de Belgo Paper Co. ${ }^{15}$. Finalement St. Maurice Valley Corporation se portait acquéreur des actifs de St. Maurice Paper Co. et des $85 \% \mathrm{du}$ capital-actions de Belgo Paper Co. moyennant l'émission de 76,000 de ses 150,000 actions ordinaires à Consolidated Investment Corpo-

\footnotetext{
12 C.B. McNaught fut administrateur de la St. Maurice Valley Corporation de 1926 à 1927.

${ }_{13}$ Archives de la Consolidated-Bathurst Co. Ltd., Minutes Book, Directors' Meeting (9 décembre 1925), document M-9-7: 51.

14 Ibid. (25 novembre 1925), document M-8-6: 43-46. L'Union Bag \& Paper Corporation vendit tous les actifs de St. Maurice Paper Company Ltd. à Consolidated Investment Corporation pour $\$ 12,139,300$. en argent liquide et 19,078 actions ordinaires de Newsprint Investment Corporation (soit $31.1 / 4 \%$ des actions ordinaires de Newsprint Investment Corporation). L'entente prévoyait aussi qu'à la réception des 19,078 actions ordinaires par Union Bag \& Paper Corporation, la société Newsprint Investment Corporation devait détenir 76,000 actions de la St. Maurice Valley Corporation. De plus la St. Maurice Valley Corporation devait avoir émis $\$ 6,800,000$. de ses $\$ 10,000,000$. en actions privilégiées rapportant $7 \%$ par année, 150,000 actions ordinaires et $\$ 8,700,000$. de ses $\$ 15,000,000$. d'obligations de première hypothèque rapportant $6 \%$ par année.

15 Cette opération fut probablement effectuée en bourse.
} 
ration et de 73,000 autres à Newsprint Investment Corporation ${ }^{16}$. Cette opération permettait au groupe Holt-Gundy le contrôle de St. Maurice Valley Corporation, contrôle auquel l'Union Bag \& Paper Corporation participait minoritairement.

Dans les années qui suivirent, les opérations du groupe HoltGundy devaient mener à la formation de Canada Power \& Paper Corporation, une société regroupant en 1928 la St. Maurice Valley Corporation et la Laurentide Co. Ltd. Dès l'automne 1927, J.H. Gundy commençait une série de négociations avec les membres du conseil d'administration de la Laurentide Co. Ltd. sur la possibilité d'une fusion avec St. Maurice Valley Corporation ${ }^{17}$. Soulignons que ces négociations ont dû être facilitées par la présence au conseil d'administration de la Laurentide de deux administrateurs de la Banque Royale, soit W.F. Robinson et J.H. Price ${ }^{18}$. D'autre part la Laurentide Co. Ltd. était depuis fort longtemps liée au groupe de la Banque de Montréal ${ }^{19}$, ayant trois administrateurs communs avec celle-ci en 1910 et en 1923 (voir tableaux 4 et 6). À la veille de la formation de Canada Power \& Paper Corporation, en 1927, C.R. Hosmer, J.W. McConnell et Sir V. Mereclith siégeaient à la fois au conseil d'administration de la Banque de Montréal et à celui de la Laurentide Co. Ltd.

L'entrée de la Laurentide Co. Ltd. au sein de Canada Power \& Paper Corporation impliquait certaines concessions de part et d'autre. J.W. McConnell et Sir V. Meredith devenaient membres du

16 Archives de la Consolidated-Bathurst Co. Ltd., Minutes Book, Directors' Meeting (9 décembre 1925), document M-9-7: 43-51. L'entente entre St. Maurice Valley Corporation et Consolidated Investment Corporation prévoyait que Consolidated Investment Corporation cédait à St. Maurice Valley Corporation 67,150 des 85,000 actions ordinaires de Belgo Paper Co. et les actifs de St. Maurice Paper Co. contre $\$ 8,699,833$. en obligations de première hypothèque rapportant $6 \%$ par année de St. Maurice Valley Corporation (soit l'émission entière), $\$ 6,800,000$. en actions privilégiées de St Maurice Valley Corporation rapportant 7\% par année, 149,000 des 150,000 actions ordinaires de St. Maurice Valley Corporation ainsi que $\$ 2,600,000$. liquide dont $\$ 5,000$. en argent comptant. De plus, St. Maurice Valley Corporation acceptait que 76,000 des 149,000 actions ordinaires cédées à Consolidated Investment Corporation soient par la suite concédées à la Newsprint Investment Corporation.

17 Comité permanent de la banque, 1934: 628. Il est intéressant de noter que J.H. Gundy et W.E. Wilder, de la société Wood, Gundy \& Co., seront administrateurs de Canada Power \& Paper Corporation de 1928 à 1932.

18 Notons que la société Price Brothers \& Co. était au moins depuis 1910 liée au groupe de la Banque Royale (voir tableaux 5 et 6 )

19 Jorge Niosi, «La Laurentide $(1887-1928)$; pionnière du papier journal au Canada», RHAF, 29, no 3 (décembre 1975): 375-415. La Laurentide Pulp Co. entrait dès 1896 dans le groupe de la Banque de Montréal avec l'accession à son conseil d'administration de W.C. Van Horne et de R.B. Angus. 


\section{TABLEAU 6}

Administrateurs communs entre les 4 sociétés papetières canadiennes les plus importantes et les sociétés formant les principaux éléments des 3 grands groupes financiers canadiens (1910)

East Canada Power \& Pulp Co.

Laurentide Paper Co. Ltd.

Price Brothers \& Co. Ltd.

Spanish River Pulp \& Paper Mills Ltd.

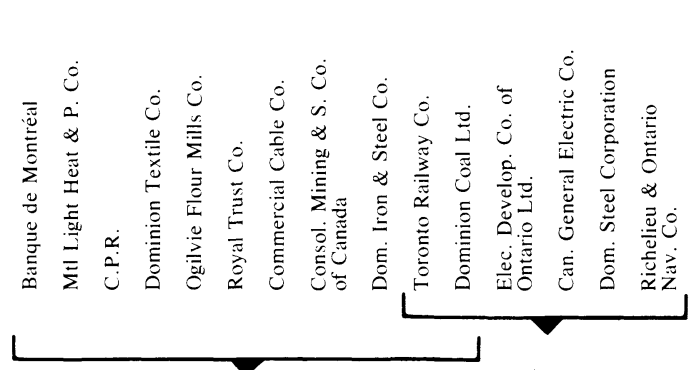

Banque de Montréal Banque Canadienne de Comm. $\begin{array}{llll}3 & 1 & 3 & 2\end{array}$

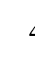

4 1 1 1 1

aucun administrateur commun)

Source: Annual Financial Review (Canadian) (Toronto, Houston’s Standard Publications) - annuel à partir de 1900-. 
conseil d'administration de Canada Power \& Paper Corporation, le premier de 1928 à 1929 et le second en 1928. Par contre le groupe de la St. Maurice Valley Corporation était représenté au conseil d'administration de Canada Power \& Paper Corporation dès 1928 par H.S. Holt, J.H. Gundy et par C.B. McNaught. La Banque de Montréal et la Banque Royale s'occupaient du compte courant de la nouvelle société. La Wood, Gundy \& Co. se chargeait de la vente de $\$ 6,000,000$. en obligations de Canada Power \& Paper Corporation rapportant $5 \frac{1}{1} 2 \%$ d'intérêt par année (émission appelée Laurentide Series) en mars 1928.

La formation de Canada Power \& Paper Corporation impliquait un échange d'actions ordinaires entre cette dernière et St. Maurice Valley Corporation et Laurentide Co. Ltd. Les conditions de l'échange d'actions furent les suivantes: les actionnaires de St. Maurice Valley Corporation recevaient $2.1 / 2$ actions ordinaires de Canada Power \& Paper Corporation d'une valeur de $\$ 40.00$ l'action, soit au total $\$ 100.00$ pour chacune de leurs actions ordinaires valant $\$ 85.00$ l'unité ${ }^{20}$. D'un autre côté les actionnaires de Laurentide Co. Ltd. obtenaient pour chacune de leurs actions ordinaires valant $\$ 108.64$ pièce, une action ordinaire $(\$ 40.00)$ et une obligation de $\$ 100.00$ (rapportant $51 / 2 \%$ d'intérêt par année) de Canada Power \& Paper Corporation, soit une valeur globale de $\$ 140.00^{21}$. Dans le cas qui nous occupe, il semble que les promoteurs aient tenu plus compte de la capacité de rapporter des dividendes des divers titres et de leur cote boursière que du rapport capital propre/actions ordinaires en circulation dans la fixation des conditions de l'échange ${ }^{22}$. Compte

20 On établit généralement la valeur des actions ordinaires d'une société en divisant la valeur de son capital propre (comprenant le montant des actions ordinaires et privilégiées en circulation, l'excédent de capital représentant le montant payé en plus de la valeur nominale ou légale des actions et enfin less bénéfices non distribués) par le nombre d'actions ordinaires en circulation. Voir Merrill Lynch, Pierce, Fenner \& Smith Inc., Comment lire un rapport financier (Montréal, 1972), 23p. - voir p. 11. A la fin de 1927, la valeur d'une action ordinaire par rapport au capital propre se chiffrait à $\$ 85.00$ pour St. Maurice Valley Corporation et à $\$ 108.64$ pour Laurentide Co. Ltd. tandis qu'elle atteignait $\$ 40.00$ à la fin de 1928 pour Canada Power \& Paper Corporation.

21 Roberta Nova McEachran, Corporate reorganizations, thèse de maîtrise ès arts (University of Toronto, 1934), 4.

22 En effet, l'action ordinaire de la Laurentide Co. Ltd. s'était vendue de $\$ 110.00$ à $\$ 139.00$ de décembre 1927 à mars 1928. Celle de Canada Power \& Paper Corporation était distribuée à un prix allant de $\$ 40.00$ à $\$ 42.00$ dans les trois premiers mois de 1928. Voir Hubert C. Dell, A financial analysis of some recent consolidations in the pulp and paper industry, thèse de maîtrise (University of Toronto, 1931), 102 (à l'avenir: H.C. Dell, A financial analysis). 
tenu du fait que les actions ordinaires de Canada Power \& Paper Corporation n'ont jamais rapporté de dividendes, les actionnaires de la Laurentide Co. Ltd., contrairement à ceux de la St. Maurice Valley Corporation, ont été favorisés dans l'échange d'actions ${ }^{23}$. Mais si les conditions de l'échange favorisaient les gens de la Laurentide Co. Ltd., le contrôle du bloc principal des actions ordinaires était entre les mains des actionnaires de la St. Maurice Valley Corporation. $43.9 \%$ des actions du bloc principal revenaient à ceux-ci tandis que ceux de la Laurentide Co. Ltd. en détenaient $25.4 \%{ }^{24}$.

En 1929, le groupe Hold-Gundy réussit à faire entrer dans la Canada Power \& Paper Corporation deux nouvelles sociétés, Wayagamack Pulp \& Paper Co. et Port Alfred Pulp \& Paper Corporation. En 1923, H.S. Holt, administrateur chez Price Brothers \& Co. Ltd., conseillait mais sans succès à Jack Price de racheter la faillite de la Bay Sulphite Co. Ltd., l'ancêtre de Port Alfred Pulp \& Paper Corporation ${ }^{25}$. J.H. Gundy pressait aussi Price Brothers \& Co., et même Abitibi Power \& Paper Co. d'acquérir Bay Sulphite Co. Ltd. Devant leur refus, J.H. Gundy l'achetait pour son propre compte $^{26}$. Élu administrateur de Bay Sulphite Co. Ltd., en 1923, J.H. Gundy la réorganisait l'année suivante, sous le nom de Port Alfred Pulp \& Paper Corporation et il en sera administrateur de 1924 à 1932. H.S. Holt occupera dans cette même société le poste d'administrateur de 1926 à 1932 ainsi que celui de président du conseil d'administration de 1925 à 1929. Quant à l'absorption de la Wayagamack Pulp \& Paper Co. Ltd., par Canada Power \& Paper Corporation, elle fut facilitée par la présence de G.H. Duggan, à la fois administrateur de la Banque Royale et de la Wayagamack Pulp \& Paper Co., de 1917 à 1932. La société Anticosti Corporation était

23 Notons de plus que les actions ordinaires de la Laurentide Co. Ltd. avaient rapporté \$6.00 par action par an pour la période allant de 1920 à 1927 inclusivement. (Ibid., 132, col. 10). Maintenant, en tenant compte des conditions de l'échange, chaque ex-actionnaire de Laurentide Co. Ltd. pouvait retirer par action échangée un revenu égal à $\$ 7.50$, soit $\$ 5.50\left(5 \frac{1}{2} \%\right.$ d'intérêt par an sur $\$ 100.00$ en obligation) plus $\$ 2.00$ (à supposer que l'actionnaire ait investi le montant de $\$ 40.00$, valeur de son action ordinaire de Canada Power \& Paper Corporation, dans l'achat d'une obligation d'une quelconque autre société rapportant 5\% d'intérêt par an). Voir ibid., 103.

24 Archives de la Consolidated-Bathurst Co. Ltd., Minutes Book, document M-8-3 (liste des 60 actionnaires les plus importants de Canada Power \& Paper Corporation), 4 pages. Le bloc principal représente 278,906 actions ordinaires, soit $40 \%$ des 688,000 actions ordinaires émises par Canada Power \& Paper Corporation au 14 août 1928. Ces 278,906 actions sont détenues par 60 actionnaires qui forment ensemble le groupe contrôlant Canada Power \& Paper Corporation.

25 Comité permanent de la banque, 1934: 785.

26 Ibid.: 623. 
depuis 1926 un point de rencontre de plus entre les futures constituantes de Canada Power \& Paper Corporation.

L'intégration de Wayagamack Pulp \& Paper Co. Ltd. et de Port Alfred Pulp Paper Corporation à Canada Power \& Paper Corporation se fit, tout comme dans le cas précédent, par un échange d'actions ordinaires entre les diverses sociétés. Les actionnaires de Port Alfred Pulp \& Paper Corporation reçurent pour chacune de leurs actions ordinaires, $23 / 4$ actions ordinaires de Canada Power \& Paper Corporation, soit $\$ 105.00(23 / 4 \times \$ 38.00)$ pour leur $\$ 122.00^{27}$. D'autre part, les actionnaires de Wayagamack Pulp \& Paper Co. Ltd. obtenaient pour chacune de leurs actions ordinaires, 1 1/2 action ordinaire et une obligation de $\$ 50.00$ (rapportant $5 \% \%$ d'intérêt par année) de Canada Power \& Paper Corporation ${ }^{28}$. Là encore, les conditions de l'échange tenaient beaucoup plus compte de la rentabilité des sociétés que de la valeur du capital-actions par rapport au capital propre. En fait l'action ordinaire de Wayagamack Pulp \& Paper Co. Ltd. avait rapporté successivement $\$ 3.75$ et $\$ 3.00$ en 1927 et en $1928^{29}$, tandis que celle de Port Alfred Pulp \& Paper Corporation n'a jamais payé de dividendes depuis sa formation en $1924^{30}$. Mais, si les actionnaires de Wayagamack Pulp \& Paper Co. Ltd. étaient favorisés par les conditions de l'échange d'actions, ceux de Port Alfred Pulp \& Paper Corporation obtenaient 21.2\% du bloc principal des actions ordinaires de Canada Power \& Paper Corporation comparativement à $14.3 \%$ pour les premiers ${ }^{31}$. Ainsi, les deux étapes de la formation de Canada Power \&: Paper Corporation (1928

27 À la fin de 1928, la valeur d'une action ordinaire de Wayagamack Pulp \& Paper Co. Ltd. par rapport à son capital propre se chiffrait à $\$ 85.00$ tandis qu'elle atteignait \$122.00 dans le cas de Port Alfred Pulp \& Paper Corporation. On évaluait la valeur d'une action ordinaire de Canada Power and Paper Corporation par rapport à son capital propre à $\$ 38.00$ au 31 décembre 1929. Voir H.C. Dell, $A$ financial analysis, 107-108.

28 Soit $\$ 2.75$ (5\% \% d'intérêt par année sur $\$ 50.00$ en obligation) plus $\$ 2.85$ (à supposer que l'actionnaire ait investi le montant de $\$ 57.00$, valeur de son $11 / 2$ action ordinaire de Canada Power \& Paper Corporation, dans l'achat d'une obligation d'une quelconque autre société rapportant $5 \%$ d'intérêt par an) donnant au total $\$ 5.60$.

29 Ibid., 133, colonne 10.

30 Ibid., 134, colonne 10.

31 Archives de la Consolidated-Bathurst Co. Ltd., list of the capital stockholders of Canada Power \& Paper Corporation, 5 septembre 1931, document M-1-10: $376 \mathrm{p}$. Le bloc principal représente 942,387 actions ordinaires, soit $62.19 \%$ des 1,515,170 actions ordinaires émises par Canada Power \& Paper Corporation au 5 septembre 1931. Ces 942,837 actions sont détenues par 36 actionnaires importants qui forment ensemble le groupe contrôlant Canada Power \& Paper Corporation. 
et 1929), profitables pour les actionnaires de la Laurentide et de Wayagamack Pulp \& Paper avaient été coûteuses pour les actionnaires de St. Maurice Valley Corporation et de Port Alfred Pulp \& Paper Corporation. Mais le groupe de la Banque Royale, avec l'aide de Wood, Gundy \& Co., réussissait à former Canada Power \& Paper Corporation et à en garder le contrôle effectif. À la fin de 1929, le groupe Holt-Gundy détenait $52.7 \%$; la Laurentide Co. Ltd. voyait sa part du bloc principal passer de $25.4 \%$ à $12.1 \%$. Le cumul des actions ordinaires de Canada Power \& Paper Corporation détenues par Laurentide Co. Ltd. et Wayagamack Pulp \& Paper Co. Ltd. représentait à peine $26.4 \%$ du bloc principal, soit un pourcentage deux fois inférieur à celui contrôlé par le groupe majoritaire de la Banque Royale.

L'objectif du groupe Holt-Gundy, en favorisant la formation de St. Maurice Valley Corporation, puis de Canada Power \& Paper Corporation, visait à empêcher la chute rapide des prix du papier journal et à limiter les investissements dans le secteur des pâtes et papiers. Les témoignages de H.S. Holt et de J.H. Gundy devant le comité permanent de la banque en 1934 en font foi ${ }^{32}$. Après 1926, aucune machine à papier n'a été installée dans les usines des sociétés du groupe Holt-Gundy ${ }^{33}$. 70\% de l'augmentation de la capacité de production de papier journal entre 1927 et 1931 était attribuable aux investissements des sociétés américaines et anglaises et se répartissait ainsi: International Power \& Paper Co. Ltd.: 21\%; Abitibi Power \& Paper Co. Ltd.: 15\%; Backus-Brooks Co. Ltd.: 14\%; Spruce Falls Power \& Paper Co. Ltd.: 10\%; Anglo-Canadian Pulp \& Paper Co.: 10\%. Durant la même période certaines sociétés canadiennes augmentaient leur capacité de production dans les proportions suivantes: St. Lawrence Paper Mills: 10.5\%; Price Brothers Co. Ltd.: 5\%; Mersey Paper Co. Ltd.: 5\% et Bathurst Co. Ltd. : $1.5 \%{ }^{34}$.

Notons en passant que le groupe de la Banque Royale a probablement favorisé l'absorption de Spanish River Pulp \& Paper Mills par Abitibi Power \& Paper Co. en 1928. L'Abitibi Power \& Paper Co. Ltd. possédait, dès 1923, trois administrateurs en commun avec la Banque Royale (voir tableau 4). De plus soulignons qu'Abitibi

32 Comité permanent de la banque, 1934: 645 (témoignage de J.H. Gundy); 788 (témoignage de H.S. Holt).

33 Comité permanent de la banque 1934: 922.

34 Ibid.: 654-655. 
Power \& Paper Co. Ltd. détenait avec Canada Power \& Paper Corporation le contrôle de la General Power \& Paper Co. à partir de $1928^{35}$.

\section{La politique expansionniste de l'International Power \& Paper Co. (1924-1930)}

La situation de surinvestissement et sa conséquence immédiate, la surproduction, sévissaient dans l'industrie canadienne des pâtes et papiers depuis la fin de la guerre de 1914-1918. Comme nous l'avons déjà souligné le prix de la tonne de papier journal s'effondra de 1923 à 1934. La Banque Royale tentait de protéger les lourds prêts qu'elle avait accordés aux diverses sociétés papetières canadiennes. Afin de maintenir les prix du papier journal elle favorisait de 1923 à 1931 la concentration des sociétés papetières canadiennes, plus particulièrement la formation successive de St. Maurice Valley Corporation en 1925 et de Canada Power \& Paper Corporation en 1928 et 1929.

Par ailleurs, les grands journaux américains étroitement liés à l'International Power \& Paper Co. Ltd. voyaient d'un mauvais œil la formation d'un cartel canadien qui entraînerait à plus ou moins brève échéance une hausse du prix du papier journal et la répétition des conditions qu'avaient connues les grands journaux américains durant la guerre: des prix excessifs pour le papier journal canadien malgré le surinvestissement et des profits plus qu'appréciables pour les sociétés canadiennes aux dépens des acheteurs américains. L'International Power \& Paper Co. Ltd. voyait son leadership dans la fixation des prix de plus en plus menacé dans une période où ses usines, situées aux États-Unis, devaient convertir leur production de papier journal en production de papiers fins, suite à l'épuisement des réserves forestières américaines et à l'abolition progressive du tarif américain sur l'importation de papier journal canadien entre 1911 et 1913.

Pour faire échec à la formation d'un cartel canadien du papier journal, l'International Power \& Paper Co. Ltd. déclara une véritable guerre des prix aux sociétés canadiennes. Sa stratégie passait par la rentabilisation de sa production de papier journal et par la diversifi-

\footnotetext{
35 Moody's Manual of Investments, Industrial Securities (New York, Analyses Publishing Co.), (voir aussi l'appendice).
} 
cation de ses revenus ${ }^{36}$. La conversion de ses usines américaines de papier journal en usines à papiers fins permettait à l'International Power \& Paper Co. Ltd. de profiter au maximum du tarif protecteur sur les papiers fins encore en vigueur aux États-Unis. Dès 1916, le tiers de sa production de papier journal avait été converti en papiers fins ${ }^{37}$. Puis venait la conversion des usines de Niagara Falls (1920) et de Fort Edward (1926). L'accaparement du plus grand nombre possible de concessions forestières dans des régions susceptibles de permettre la production de papier journal à bon compte représentait un autre aspect de l'effort de rentabilisation de l'International Power \& Paper Co. Ltd. De 1925 à 1928 cette société se lança dans l'acquisition massive de terres à bois tant aux États-Unis qu'au Canada: 1925, obtention de 376 milles carrés de terres de la Couronne en Gaspésie pour l'usine de Trois-Rivières et de 2,000 milles carrés de terres de la Couronne dans le bassin supérieur des rivières Ottawa et Gatineau pour les usines de Kipawa et de Gatineau; 1925 et 1926, concession de 615 milles carrés de terres de la Couronne dans les bassins des rivières Restigouche et Nouvelle pour l'usine du Nouveau. Brunswick; 1926, mainmise sur 584 milles carrés de terres privées et signature d'une promesse d'achat pour 103 autres milles carrés de boisés en Louisiane et en Arkansas pour sa nouvelle filiale, la Southern International Paper Co. Ltd.; 1927, obtention de 1,210 milles carrés de terres de la Couronne au Nouveau-Brunswick, de Nipigon Corporation (une société détenant 1,568 milles carrés de terres de la Couronne en Ontario) et de 168 milles carrés en Arkansas; 1928, addition de 2,707 milles carrés de boisés à TerreNeuve, de 745 milles carrés de terres de la Couronne au Québec et de 66 milles carrés pour l'usine de Moss Point au Mississipi.

La rentabilisation de la production de papier journal se traduisit aussi par la construction d'usines à papier journal à haut rendement aux États-Unis et au Canada: 1924, installation de deux nouvelles machines à Trois-Rivières pouvant doubler la capacité de production de l'usine; 1925, achat de la société canadienne Riordon Co. Ltd., début de la construction d'une usine de papier journal sur la rivière Gatineau (achevée en 1927), capacité de production de l'usine à pâte sulphite de Kipawa doublée (ex-usine de la Riordon Co. Ltd.) et

36 H.C. Dell, A financial analysis, 48.

37 Les diverses informations touchant les multiples aspects de la stratégie de l'International Power \& Paper Co. Ltd. sont tirées du Moody's Manual of Investments Industrial Securities (voir aussi l'appendice). 
acquisition du capital-actions de Bastrop Pulp \& Paper Co. (une usine à papier Kraft en Louisiane à laquelle on ajoute une nouvelle machine); 1926, installation d'une deuxième machine à Bastrop Pulp \& Paper Co. Ltd. ; 1927, formation de la filiale International Power \& Paper Company of Newfoundland Ltd. pour acquérir l'usine de Corner Brook, achat d'une des usines de la Louisiana Pulp \& Paper Co., construction d'un moulin à Camden en Arkansas, formation de la filiale Southern International Paper Co. (englobant les usines de Bastrop, de Camden et celle achetée à la Louisiana Pulp \& Paper Co. Ltd.), achat des usines américaines de De Grasse, St. George, High Falls et Norwalk, début de la production à sa filiale Veldon Inc., achat des dernières propriétés de la Louisiana Pulp \& Paper Co. Ltd.; 1928, début de la construction de l'usine de Dalhousie au NouveauBrunswick (qui commencera à produire en 1929), achat d'une usine à Moss Point au Mississipi et démarrage d'une nouvelle usine à Mobile en Arkansas; 1930, deux nouvelles machines entrent en production à Dalhousie, Nouveau-Brunswick. Toute cette politique de rentabilisation de la production de papier journal menée par l'International Power \& Paper Co. Ltd. permettait des économies substantielles et une guerre des prix qui ne pouvait que nuire aux sociétés du groupe Holt-Gundy.

La diversification des revenus de l'International Power \& Paper Co. Ltd. formait l'autre volet de sa stratégie. La société effectua de 1925 à 1930 d'énormes investissements dans le secteur hydro-électrique tant au Canada qu'aux États-Unis: 1924, entrée en opération du Sherman Island Development, du barrage Feeder sur la rivière Hudson et de la centrale de sa filiale, Moreau Manufacturing Co. Ltd.; 1925, construction sur des sites non-exploités de l'ex-Riordon Co. Ltd. de deux centrales hydro-électriques (celles de Chelsea et de Farmer's Rapids) ainsi que d'une digue à Bitoli Rapids afin de régulariser la rivière Gatineau, acquisition d'intérêts substantiels dans New-England Power Association; 1926, achat au Canadian Pacific Railway du site inexploité de Paugan, formation de la filiale Gatineau Power Co. pour gérer les sites de Chelsea, Farmer's Rapids et de Paugan, signature d'un contrat de vingt ans entre Gatineau Power Ltd. et l'Hydro Electric Power Commission of Ontario pour la livraison de 1,120 kilowatts/heure moyennant \$3.9 millions par année à partir du premier octobre 1928, acquisition du capital-actions de Hull Electric Co., Papineauville Electric Co., Bonhomme Hydro-Electric Development \& Distributing System et de Maniwaki Power \& Telephone Co., début de la construction d'une centrale hydro-électrique 
à Grand-Falls sur la rivière Saint-Jean par sa filiale St. John River Power Co., parachèvement de la centrale de Kipawa, réfection du barrage de l'usine de Wilder sur la rivière Connecticut et de la digue de l'usine de Webster sur la rivière Penobscot, début de la construction d'une centrale à vapeur pour l'usine Otis de Livermore Falls (Maine), achat d'un nouveau bloc d'actions dans New England Power Association; 1927, formation de la filiale Canadian Hydro-Electric Corporation (englobant Gatineau Power Co., Gatineau Electric Light Co. et St. John River Power Co.), signature d'un autre contrat de 10 ans avec l'Hydro-Ontario entrant en vigueur le premier octobre 1928, formation de sa filiale Gatineau Electric Co. pour gérer OttawaMontreal Power Co., Quebec Southern Power Corporation, Laurentide Hydro-Electric Ltd., Papineauville Electric Co. et Maniwaki Power \& Telephone, achat d'un autre bloc d'actions portant à 45 le pourcentage d'actions qu'elle détenait dans New England Power Association; 1928: livraison par sa filiale Gatineau Power d'énergie électrique à Toronto conformément au contrat signé avec l'HydroOntario, la Gatineau Power Co. acquiert Ottawa River Power Co. et Ottawa \& Hull Power Co., première livraison d'énergie électrique de la St. John River Power Co. à la société Fraser Companies. En 1929, l'International Power \& Paper Co. créait sa filiale International Hydro-Electric System, une société de gestion contrôlant Canadian Hydro-Electric Corporation et le bloc d'actions qu'elle détenait dans New England Power Association. À la fin des années 1920, l'International Power \& Paper Co. était devenue le plus grand producteur de papier et d'électricité au monde ${ }^{38}$.

L'International Power \& Paper Co. Ltd., conformément à sa politique de diversification de ses revenus, investit aussi dans certains produits connexes comme le contre-plaqué, les cartonnages et l'imprimerie: 1927, formation de sa filiale International Fibreboard Ltd. (société créée pour acquérir l'usine ontarienne de Midland et pour construire une nouvelle usine près de Gatineau), achat du capital-actions de la George \& Sherrard Paper Co. de Wellsbury, Virginie de l'Ouest (conversion de cette usine de corde en usine de sacs); 1928, renouvellement d'un contrat avec les postes américaines pour la fabrication d'enveloppes affranchies et l'emballage des journaux, formation de sa filiale International Envelop Corporation en vue de l'acquisition de Middle West Manufacturing Co. de Dayton en Ohio et finalement la prise en main de Continental Paper \& Bag Corporation.

38 H.C. Dell, A financial analysis, 20. 
La politique de diversification des revenus de l'International Power \& Paper Co. aboutissait au tableau suivant en 1930: 55\% de ses revenus venaient de l'hydro-électricité, $25 \%$ de la vente de papier journal et $20 \%$ de sources diverses ${ }^{39}$. La société possédait 38 centrales hydro-électriques, 3 centrales électriques à gaz, 4 réservoirs de régularisation, 2 usines de contre-plaqué, 1 usine d'enveloppes, 44 scieries, 13 usines d'écorçage, 2 ateliers de réparation, une mine de charbon, 3 bateaux à vapeur et 4 autres usines ${ }^{40}$. Si en Amérique du Nord la situation de l'industrie papetière se dégradait rapidement à la fin des années 1920, celle de l'industrie hydro-électrique se maintenait facilement. La New England Power Association, filiale de l'International Power \& Paper Co. enregistrait une hausse de ses revenus de 1928 à $1929^{41}$. Malgré la chute des valeurs papetières, l'International Power \& Paper Co. marquait des points en bourse grâce à la tendance à la hausse des titres hydro-électriques, titres dont elle était une grande émettrice ${ }^{42}$. La diversification de ses revenus compensait aussi un endettement élevé vis-à-vis de ses obligataires ${ }^{43}$.

\section{La guerre du papier et l'échec du cartel canadien, 1921-1931}

Entre 1900 et 1911 plusieurs provinces canadiennes, dont le Québec et l'Ontario, avaient imposé des embargos successifs sur les exportations de bois à pâte coupé sur les terres de la Couronne. Les États-Unis avaient répondu par la hausse progressive de leur tarif sur le papier importé, au désespoir des grands journaux américains. La lutte de ceux-ci contre ces hausses périodiques du tarif aboutit à sa réduction en 1911 et à son abolition par l'Underwood Act en 1913, victoire qui leur permettait de s'approvisionner en papier journal bon marché. Par ailleurs les producteurs canadiens devenaient des concurrents redoutables pour les manufacturiers américains de papier journal qui faisaient alors face à une situation difficile comme nous l'avons indiqué plus haut. Certains grands producteurs américains installaient des filiales au Canada tandis que les sociétés papetières canadiennes connaissaient une expansion sans précédent. Bientôt les grands journaux américains, triomphants en

\footnotetext{
39 Ibid., 48.

Ibid., 57.

Ibid., 52.

Ibid., 51 .

Ibid., 58.
} 
1913, se mirent à déchanter. Les producteurs canadiens profitèrent de la guerre pour imposer des prix excessifs à leurs principaux clients malgré le surinvestissement général dans cette industrie. L'effort du groupe de la Banque Royale pour former un cartel de producteurs canadiens afin de contrer la baisse du prix du papier journal suite au surinvestissement dans le secteur de 1911 à 1926 ne pouvait qu'indisposer les grands journaux américains. Les difficultés éprouvées par les producteurs américains depuis 1911 les avaient menés à une intégration de plus en plus poussée aux grands journaux. S'appuyant sur ceux-ci, l'International Power \& Paper Co. Ltd. développait une stratégie pour contrôler le marché nord-américain du papier journal. Cette stratégie était fort simple: aggraver la situation de surproduction en augmentant sa propre capacité de production, provoquer par le fait même une chute du prix du papier journal, mettant ainsi les producteurs canadiens déjà lourdement endettés au bord de la faillite, se prémunir contre l'effondrement des prix par une vaste diversification de ses revenus et par l'acquisition et la construction d'unités de production à haut rendement.

La guerre de prix qui allait s'engager dans le secteur des pâtes et papiers au cours de la décennie avait pour enjeu le contrôle du prix du papier journal à l'est des Montagnes Rocheuses. Plusieurs producteurs canadiens de pâtes et papiers s'étaient regroupés de 1912 à 1921 dans des sociétés de vente, celles-ci devant faciliter l'écoulement du produit sur le marché américain et entretenir des relations suivies avec les clients. G.H. Mead Company de Dayton en Ohio s'occupait dès 1912 de vendre la production de la Spanish River Pulp \& Paper Mills et celle de l'Abitibi Power \& Paper Company à partir de 1914. En 1916, Price Brothers Co., Brompton Pulp \& Paper Co. et Laurentide Co. Ltd. formaient leur société de vente commune, la Canadian Export Co. En 1921, la St. Maurice Paper Co. et la Belgo Canadian Pulp Co. créaient respectivement leur propre société de vente, la St. Maurice Sales Co. et la Belgo Export Co. Les premiers effets de la pénétration du groupe de la Banque Royale dans le secteur des pâtes et papiers se manifestaient en 1925 par la formation de la St. Maurice Valley Corporation et en 1926 par la création de sa société filiale, la St. Maurice Valley Sales Corporation. Cette dernière société de vente, née de la fusion de St. Maurice Sales Company et de Belgo Export Co., organisait la vente de la production de trois autres sociétés importantes (St. Lawrence Paper Mills Ltd, Beaver Board Co. Ltd. et Port Alfred Pulp \& Paper Co.). 
Mais l'International Paper Co., profitant de l'endettement progressif des compagnies canadiennes, faisait passer le prix du papier journal de 84 à 56 dollars (constants) la tonne de 1921 à 1928. La Banque Royale intervenait et organisait en 1927 la Canadian Newsprint Company. Cette société de vente regroupait la St. Maurice Sales Corporation, la Canadian Export Co. et G.H. Mead Co. La Canadian Newsprint Co. devait s'occuper de la vente de la production de sept sociétés (Laurentide Co. Ltd., Price Brothers Co., Port Alfred Pulp \& Paper Co., St. Maurice Valley Corporation, Canada Paper Co., Ste Anne Paper Co. et Spanish River Pulp \& Paper Co.). Elle devait écouler leurs produits, contrôler et répartir la production et fixer les prix. À la fin de 1927, cette société tenta pour la première fois de s'opposer à l'International Paper Co. et de conclure une entente avec Hearst Publishing Co., pour une durée de dix ans, fixant le prix à $\$ 65$. la tonne de papier journal. Mais au début de 1928, la Canadian Newsprint Co. décida de ne pas exécuter le contrat. Hearst signa alors des ententes séparées avec deux sociétés associées à Canadian Newsprint Co. à 64 dollars la tonne pour les six premiers mois de 1928 et à 61 dollars pour les six derriers mois ${ }^{44}$. La Canadian Newsprint Co., incapable de discipliner ses membres, fut dissoute à la fin de 1928. Aussitôt après, les producteurs canadiens se réorganisaient. H.S. Holt formait la Canada Power \& Paper Corporation et favorisait la formation de l'Abitibi Power \& Paper Company. Avec l'appui des premiers ministres Taschereau clu Québec et Ferguson de l'Ontario, quatorze sociétés canadiennes de pâtes et papiers se regroupaient dans le Newsprint Institute of Canada. Cette union monopolistique contrôlait $70 \%$ de la production canadienne de pâtes et papiers, vingt points de pourcentage de plus que la Canadian Newsprint Co.

International Paper Co. annonçait à la fin de 1928 qu'elle avait fixé avec Hearst le prix du papier journal à un prix inférieur de sept à dix dollars. Le premier ministre Ferguson avertissait l'International Paper Co. que son gouvernement ne permettrait pas l'effondrement de ce secteur de l'économie. Taschereau annonçait une augmentation des droits de coupe pour toute société qui accepterait un prix inférieur à celui du Newsprint Institute of Canada. L'American Newspaper Publishers Association dénonçait le cartel canadien. La

44 H. Marshall, F.A. Southard, K.W. Taylor, Canadian-American Industry (New York, Russell \& Russell Co., 1936), 49 (à l'avenir, H. Marshall, CanadianAmerican Industry). 
lutte se poursuivit de décembre 1928 à février 1929. Enfin une entente survint et le Canadian Newsprint Institute obtint une augmentation de cinq dollars la tonne de papier journal. Mais le succès de l'Institute fut bref. Dès la fin de 1929, Graustein, le président de l'International Paper Company, refusa catégoriquement une autre augmentation de cinq dollars la tonne réclamée par le Canadian Newsprint Institute pour 1930. L'union monopolistique du Canadian Newsprint Institute of Canada s'effondra en 1930, laissant les producteurs canadiens complètement désorganisés.

En octobre 1930, les producteurs canadiens, toujours groupés autour de la Banque Royale, proposaient à l'International Paper Co. la constitution d'une union géante. L'International se montra trop exigeante et la tentative échoua ${ }^{45}$. La faillite guettait les sociétés canadiennes. Les sociétés liées au groupe de l'International Paper Co. qui opéraient au Canada avaient maintenu de 1929 à 1933 une production remarquablement stable ${ }^{46}$. Les liens étroits qu'elles entretenaient avec les grands journaux américains leur permirent de passer tant bien que mal à travers la crise ${ }^{47}$. Les sociétés du groupe de la Banque Royale virent diminuer leur production de 700,000 tonnes de papier journal de 1929 à 1933. Le groupe de la Banque Royale avait subi le plus gros de la dépression. Il avait perdu la partie. Canada Power \& Paper Corporation, Abitibi Power \& Paper Co., Price Brothers \& Co., St. Lawrence Corporation et Backus-Brooks Co., représentant $58 \%$ de la capacité canadienne de production, étaient acculées à la faillite. De 1931 à 1939 plusieurs sociétés durent être réorganisées. Les actions de la Canada Power \& Paper Corporation subissaient une dévaluation de $90 \%$, passant de dix dollars l'action en 1929 à un dollar en $1931^{48}$. Pour 10 actions de Canada Power \& Paper Corporation les actionnaires recevaient une action de la nouvelle Consolidated Paper Corporation. Hearst Publishing Co. de New-York, acquérait au cours de cette opération des intérêts prépondérants dans Consolidated Paper Corporation ${ }^{49}$. Donnacona

45 J.A. Guthrie, The Newsprint Paper Industry, an economic analysis (Cambridge, Harvard University Press, 1941), 67 (à l'avenir: J.A. Guthrie, The Newsprint Paper Industry).

46 H. Marshall, Canadian-American Industry, 52.

47 J.A. Guthrie, The Newsprint Paper Industry, 72. 1950), 42.

48 P.C. Newman, The Flame of Power (Toronto, Longmans Green \& Co.,

A. Faucher, "Pouvoir politique et pouvoir économique dans l'évolution du Canada français ", Albert Faucher, Histoire économique et unité canadienne (Montréal, Fides, 1970), 191. 
Paper Co. diminuait de $40 \%$ la valeur de ses titres en $1932^{50}$. En avril 1936 Brompton Pulp \& Paper Co. réduisait la valeur de son capital fixe de 50\% ${ }^{51}$. L'Abitibi Power \& Paper Co. se réorganisait en 1939.

\section{Conclusion}

On peut attribuer l'échec de la tentative de formation d'un cartel canadien par le groupe de la Banque Royale à plusieurs facteurs. Le premier fut évidemment le surinvestissement dans le secteur et son corollaire, le fort endettement des sociétés papetières canadiennes. Nous avons déjà examiné un peu plus haut leur endettement auprès des banques. Soulignons de plus que le type même de financement employé lors des diverses fusions et concentrations accentuait un endettement déjà lourd. Notons par exemple qu'en 1929 Canada Power \& Paper Corporation consacrait $48.66 \%$ de ses revenus au seul paiement des intérêts sur les obligations émises ${ }^{52}$. Ce même pourcentage s'élevait à $37.30 \%$ dans le cas de l'Abitibi Power \& Paper Co. comparativement à $30.47 \%$ dans celui de l'International Power \& Paper Co. ${ }^{53}$ La diversification des revenus de l'International Power $\&$ Paper Co. Ltd. demeure un facteur important de son succès. Ses investissements massifs dans le secteur de l'électricité lui assuraient un revenu stable et de plus en plus important à mesure que ses contrats entraient en vigueur. Cette société était devenue un des plus grands distributeurs et producteurs d'électricité en Amérique du Nord. Les plans de développement hydro-électrique de la société Abitibi Power \& Paper Co. restaient modestes tandis que de son côté Canada Power \& Paper Corporation vendait Laurentide Power Co. en $1928^{54}$. International Power \& Paper Co. produisait une grande variété de produits comme du bois de construction, du papier journal, du papier d'emballage (papier kraft), des sacs, des enveloppes, des papiers fins, des cartonnages, des allumettes, etc... Canada Power \& Paper Corporation se contentait de produire du papier journal et un peu de papier kraft à son usine de Trois-Kivières. Abitibi Power \& Paper Co. tentait de diversifier sa production en achetant en 1930 Provincial Paper Mills Co., une société produisant des papiers fins mais surtout du papier kraft.

\footnotetext{
50 J.A. Guthrie, The Newsprint Paper Industry, 67.

51 Ibid., 68.

52 H.C. Dell, A financial analysis, 128, colonne 7.

53 Ibid., 125, 126, colonne 7.

54 Ibid., 116.
} 
Il semble aussi que la répartition géographique des usines de l'International Power \& Paper Co., disséminées de Terre-Neuve à la Louisiane, favorisait cette dernière du seul point de vue des coûts de transport et de distribution. L'Abitibi Power \& Paper Co. avait réparti ses usines d'est en ouest, de Murray Bay sur le Saint-Laurent au cœur du Manitoba. Canada Power \& Paper Corporation, avec ses usines concentrées surtout dans la région de Trois-Rivières, occupait probablement une situation moins propice aux échanges. Mais il ne faudrait pas surestimer ce facteur.

Ce qui ressort le plus clairement, c'est la dépendance presqu'entière des producteurs canadiens de papier journal envers le marché américain pour écouler leur produit. De plus, le renforcement des liens entre les grands journaux américains et certains producteurs américains importants comme l'International Power \& Paper Co. Ltd. limitait grandement les possibilités de vente des producteurs canadiens en temps de crise. Dans une situation de surproduction chronique, endettées lourdement auprès des banques et des obligataires, face à un marché de plus en plus exigü, les sociétés papetières canadiennes avaient du mal à s'organiser et l'effort du groupe de la Banque Royale pour former un cartel solide se solda par un dur échec. L'International Power \& Paper Co. Ltd. conserva le contrôle des prix et les grands journaux américains s'assuraient une source de papier journal peu coûteux. 


\section{APPENDICE}

\section{Abitibi Power \& Paper Co., Canada Power \& Paper Corporation et International Power \& Paper Co. et leurs filiales en 1930}

1. Abitibi Power \& Paper Co. (1914)

Profits bruts: 7.4 millions de dollars

Capacité de production: 2,600 tonnes par jour

Ses filiales

1914 - Abitibi Pulp \& Paper Co. (1912)

1928 - Manitoba Paper Co.

Pine Falls Power Co.

1928 - Sainte Anne Paper Co.

1927 - Mattagami Pulp \& Paper Co.

1928 - Spanish River Pulp \& Paper Mills (1910) 1910 - Spanish River Pulp \& Paper Co. (1899)

1912 - Ontario Pulp \& Paper Co.

1913 - Lake Superior Paper Co.

1928 - Fort William Paper Co.

1928 - Fort William Power Co. Ltd.

1928 - Murray Bay Paper Co.

1928 - (acquiert 50\% du capital-actions de) General Power \& Paper Co. Thunder Bay Paper Co.

1930 - Provincial Paper Co.

Provincial Paper Sales Ltd.

1930 - Ontario Power Service Corporation Ltd.

Hudson's Bay Power Co.

1930 - Kaministiquia Power Co.

1930 - Iroquois Falls Drug Co.

Mattagami Railroad Co.

2. Canada Power \& Paper Corporation (1928)

Profits bruts: $\quad 6.2$ millions de dollars

Capacité de production: 3,200 tonnes par jour

Ses filiales

1928 - (contrôle 50\% du capital-actions de) General Power \& Paper Co. Ltd.

1928 - St. Maurice Valley Corporation (1925) 
1925 - St. Maurice Paper Co. (1915)

1915 - Charlemagne \& Lac Ouareau Lumber Co.

1915 - Grès Falls Co.

1915 - St. Gabriel Co. Ltd.

1915 - St. Maurice Securities (1915)

1921 - St. Maurice Sales (1921)

1925 - St. Maurice Valley Paper Co. Ltd. (1925)

1925 - Belgo Paper Co. (1922)

1922 - Belgo Pulp \& Paper Co. (1904)

1904 - Belgo Canadian Pulp Co. (1900)

1921 - Belgo Export Co. (1921)

1926 - St. Maurice Valley Sales Corporation (1926)

1926 - (contrôle le tiers du capital-actions de) Anticosti Corporation (1926)

1926 - Anticosti Shipping Co. (1926)

1926 - Canada Paper Co. (1873)

1927 - Canada Newsprint Co. (1927)

1928 - (contrôle en partie avec Building Products Ltd.) National Forest Products Ltd.

1928 - Laurentide Co. (1911)

1911 - Laurentide Paper Co. (1903)

1903 - Laurentide Pulp Co. (1887)

Canada Pulp Co. (1882) (liquidée en 1883)

1915 - Laurentide Power Co. (1915)

1927 - Laurentide-Ottawa Co. (1927)

1927 - Menjobagues Lumber Co. (1920)

1927 - la Seigneurie Perthuis

1929 - Port-Alfred Pulp \& Paper Co. (1924)

1924 - Bay Sulphite Ltd. (1922)

1922 - Ha Ha Bay Sulphite Co. (1916)

1926 - (contrôle le tiers du capital-actions de) Anticosti Corporation (1926)

1926 - Anticosti Shipping Co. (1926)

1927 - (contrôle 50\% du capital-actions de) Quebec Pulp \& Paper Mills Ltd. (1927)

1929 - Wayagamack Pulp \& Paper Co. (1910)

1924 - Wayagamack News Co. (1924)

1926 - (contrôle le tiers du capital-actions de) Anticosti Corporation (1926)

1926 - Anticosti Shipping Co. (1926)

1930 - Anglo-Canadian Pulp \& Paper Mills Ltd. (1924) 
3. International Power \& Paper Co. (1928)

Profits bruts: $\quad 41.3$ millions de dollars

Capacité de production: 4,000 tonnes par jour.

Ses filiales:

A) 1928 - I'International Paper Co. (1898)

1925 - Canadian International Paper Co. (1925)

1925 - Canadian International Paper Co. (1916)

1916 - St. Maurice Lumber (1891)

1925 - Riordon Co. Ltd.

1928 - Bathurst Power \& Paper Co. (1928)

1928 - Bathurst Co. Ltd. (1907)

Bathurst Electric \& Water

Power Co. Ltd.

1928 - Continental Paper \& Bag Corporation (1928)

Continental Paper Products Ltd.

Continental Wood Products Ltd.

Falls Manufacturing Co.

Union Falls Power Co.

Marinette \& Menominee Paper Co.

Oconto River Improvement Co.

York Haven Paper Co.

Grand Lake Co.

Victory Bay \& Paper Co.

Kapuskasing River Improvement Co.

Ticonderoga Pulp \& Paper Co.

1928 - International Fibreboard Ltd.

Veldon Co. Inc.

George \& Shenard Paper Co.

Nipigon Corporation

1928 - New Brunswick International Paper Co.

Boston Publishing Co. (contrôlée à 50\%)

International Securities Co

1929 - International Power \& Paper Securities Inc.

1929 - International Power \& Paper Securities Ltd.

Holed Tite Corporation (contrôlée à 50\%)

1930 - Waterway Paper Products Co.

B) 1929 - International Power \& Paper Co. of Newfoundland Ltd. (1927)

Newfoundland Power \& Paper Co.

C) 1929 - International Hydro-Electric System (1929)

1929 - Canadian Hydro-Electric Corporation Ltd. (1927)

Gatineau Power Co. (1926)

Gatineau Transmission Co. 
St. John River Power Co.

St. John River Storage Co.

Hawkesbury Electric Light \& Power Co.

Electric Light Co. Ltd.

Gatineau Electric Light Co. Ltd.

1929 - New England Power Association

Bellows Falls Hydro-Electric Corp.

Fall Mountain Electric Co.

Connecticut River Development Co.

The Connecticut River Power Co. of New-Hampshire

Connecticut Valley Company

The Deerfield Company

Deerfield River Power Company

Grafton Co. Electric Light \& Power Co.

Fall River Electric Light Co.

Hartford Water Company

Lawrence Gas and Electric Co.

The Lowell Electric Light Corp.

New England Power Co.

New England Power Construction Co.

Power Construction \& Engineering Inc.

Quiney Electric Light and Power Co.

Rhode Island Power Transmission Co.

The Rhode Island Public Service Co.

Seekonk Electric Co.

Sherman Power Construction Co.

Webster and Southbridge Gas and Electric Co.

The Worcester Electric Light Co.

New Salem Electric Co.

United Electric Railway Co.

The Narragansell Electric Co.

South County Public Service Co.

The Mystic Power Co.

East Greenwich Electric Co.

New England Power Corp. of Vermont

Sea View Railroad Co.

Bristol County Gas and Electric Co.

Providence Steam Co.

Connecticut Valley Lumber Co.

Upper Connecticut River and Lake Improvement Co.

Essex Company

Monroe Water Power Co.

Grafton Power Co.

The Caledonia Power Co.

Grafton Caledonia Power Co. 
Attleboro Steam and Electric Co.

Tiverton Electric Light Co.

Gardner Electric Light Co.

Salem Electric Light Company

South Vernon Electric Light Company

Deerfield River Electric Company

Vernon Electric Light Company

New England Power Engineering \& Service

Corporation.

Source: Les dates mises entre parenthèses qui suivent le nom des différentes filiales indiquent l'année de l'incorporation. D'autre part, les dates qui précèdent le nom des filiales indiquent l'année de l'achat ou de l'absorption de celles-ci par la société-mère. Survey of Corporate Securities, publié annuellement depuis 1928 par le Financial Post, à Montréal; Moody's Manual of Investments, Industrial Securities (publié annuellement depuis 1906) (New York, Analyses Publishing Co.) 